\title{
Urgency as the cardinal symptom of overactive bladder: a critical analysis
}

\author{
Linda Cardozo $\cdot$ Chris Chapple $\cdot$ Alan Wein
}

Received: 12 June 2009 / Accepted: 16 June 2009 / Published online: 15 November 2009

(C) Springer-Verlag 2009

Urgency, as defined by the International Continence Society is a "sudden compelling desire to pass urine which is difficult to defer" [1]. It is the cardinal symptom of the overactive bladder syndrome and is the only symptom which is required to describe an individual as suffering from an overactive bladder. This symptom complex is common amongst men and women of all ages and large epidemiological studies both in Europe and the USA suggest that there is an increasing prevalence with increasing age in both sexes and that urgency may be the most bothersome symptom for those who suffer from an overactive bladder (OAB) [2-4].

Unfortunately urgency is difficult to define and to measure both in clinical trials and clinical practice and it is often difficult to differentiate between the normal sensation of "urge" (desire) to void which is common to everyone and the symptom of "urgency" which is regarded as being pathological. Reviewing the existing literature in 2005 Chapple and colleagues [5] showed that the methods of assessing urgency were deficient in accuracy, largely unvalidated and inconsistent with the accepted ICS definition. Currently there is enormous controversy over the applica-

L. Cardozo $(\bowtie)$

Urogynaecology, King's College Hospital,

8 Devonshire Place, London W1G 6HP, UK

e-mail: 1cardozo@compuserve.com

\section{Chapple}

Sheffield Teaching Hospitals NHS Foundation Trust, Sheffield S10 2JF, UK

e-mail: c.r.chapple@shef.ac.uk

\section{A. Wein}

University of Pennsylvania School of Medicine,

9 Penn Tower, 3400 Spruce St, Philadelphia, PA 19104, USA

e-mail: awein@urotoday.com bility and usefulness of the term 'urgency', particularly as its differentiation from urge does not translate into the majority of languages. The measurement of the duration of the period from onset of urgency to voiding or incontinence (warning time [6]) has been the subject of much study and discussion and currently is not widely accepted as being a realistically reproducible measure. In addition there is some degree of scepticism at a regulatory level as to the robustness of the current criteria used to define and measure 'urgency'.

Consequently there has been increased contemporary interest in the concept of urgency as a pathological symptom and a wide range of both subjective and objective measures have been employed to try to define and measure the symptom, and evaluate its response to treatment. Recent studies have characterised urgency using objective measures such as cystometry [7] and looked at the role of subjective measures using questionnaires to try and capture sensation [8].

This supplement is largely based on the outcome of a meeting of experts convened specifically to identify what we know about urgency in contemporary practice, what gaps in our knowledge need to be filled on how we interpret and relate the results of randomised clinical trials to every day real life clinical practice.

Michel and Chapple [9] have described the basic mechanisms of underlying the genesis of urgency as it is perceived during bladder filling. They have looked at the underlying concepts and hypotheses relating to the aetiopathophysiology of detrusor overactivity and have tried to differentiate what is known about the normal sensation of bladder filling as contrasted to the pathological symptom of urgency. They have then gone on to look at the effect of various anti-muscarinic medications on the symptom of urgency, based on the results of large clinical trials. 
Whilst it is a commonly held perception that the overactive bladder syndrome is more prevalent in women than in men this is probably as a result of the "bothersomeness" of urgency incontinence which occurs much more frequently in women than in men. However lower urinary tract symptoms (LUTS) as a whole and particularly storage symptoms such as urgency are highly prevalent especially in older men. Yamaguchi et al. [10] have devoted their contribution to the place of overactive bladder in male lower urinary tract symptoms. They report that whilst historically the management of male lower-urinary tract symptoms has been dominated by a focus on the perceived obstructive component consequent upon benign prostatic hyperplasia with alpha-1 adreno-receptor antagonists being the mainstay of non-surgical management. It is clear in 2009 that there is increased recognition that the storage symptoms of $\mathrm{OAB}$ are more bothersome than previously recognised and that they need to be evaluated and treated accordingly. Furthermore they have reviewed recent studies which suggest that anti-muscarinic therapy alone or in combination with an alpha-1 adreno-receptor antagonist can improve symptoms of an overactive bladder in men with or without bladder outlet obstruction (although long terms results are unknown) and postulate that phosphodiesterase inhibitors may be potentially useful pharmacotherapy for men with LUTS.

An interesting concept is the relationship between the bladder pain syndrome (previously known as interstitial cystitis) and whether the sensation of urgency in relation to this can ever overlap with the symptom of "pain". This is explored by Hanno et al. [11]. The three symptoms of pain, frequency and urgency define the bladder pain syndrome/ painful bladder syndrome but it is likely that the urgency that is experienced by these patients is different from the sensation of urgency described by those with the overactive bladder syndrome, as a result of involuntary detrusor contractions/detrusor overactivity. Clearly further research into the pathophysiology of urgency and the associated underlying disease processes is required both in order to improve diagnosis and therefore treatment of these conditions and before more definitive conclusions can be reached.

It is acknowledged that the symptom of urgency becomes more prevalent and becomes particularly bothersome in the elderly population. Yoshida [12] has focused on the multifactorial nature of the overactive bladder in the elderly and has shown that both lifestyle interventions and pharmacological agents can be successful in treating these symptoms in elderly men and women. He has highlighted the increasing importance of this condition and the impact that this will have on society with the demographic shift towards increasing age of the population and therefore the need to raise awareness amongst healthcare professionals.
Dmochowski et al. [13] have explored the current methods of measuring the symptom of urgency and its severity including its impact on quality of life. They have included the use of bladder diaries, body maps of urgency and measuring urinary sensation during filling cystometry. They have tried to capture the multi-dimensional nature of the symptom of urgency and interpret these measurements for use in clinical practice.

The management of urgency in patients with the overactive bladder syndrome has been considered. In view of the fact that urgency is the key symptom of the overactive bladder syndrome this is a relatively new concept. Traditionally both in clinical trials and also in clinical practice the symptoms of urinary incontinence and urinary frequency both day and night have been employed to assess the efficacy of treatment and this has not always addressed the individuals need to control the troublesome symptom of urgency and the assessment of this in the context of patient reported outcome measures.

The supplement concludes with a "call to action" by Staskin [14] who has written a provocative piece regarding the need to measure urgency and differentiate it from the normal urge or desire to void. He has outlined the areas of agreement and disagreement amongst the experts involved in reviewing the potential validity of focusing on and trying to assess urgency. He has suggested the possibility of "revisiting" the definition of urgency, the value of advancing research in this area and the application of our current clinical trials in to daily clinical practice.

As editors of this supplement we are deeply indebted to all of the expert contributors all of whom were actively involved and participated in this meeting of experts. Whilst the focus here has been urgency and clearly there is a great deal of interest in this, time will tell whether refinements of assessment for urgency will actually prove effective or whether ultimately the global assessment of patients' perceptions on improvement will be just as or more useful and valid in the clinical setting. All of the manuscripts have been written totally independently from the sponsor who provided an unrestricted educational grant to support both the meeting and editorial collation and production of the supplement.

Conflict of interest statement Professor Linda Cardozo has during the last year received funding for research, lecturing and/or advice/ consultancies from the following organizations: Astellas: Member of Global advisory board for Solifenacin, co-chairman of the European overactive bladder forum, principle investigator for the SUNRISE study, speaker at satellite symposia. Pfizer: Member of Global Advisory Board for Fesoterodine, participant in a multicentre research study. Schering Plough: Gynaecological Advisory Board. Research consultancy and/or advisory work for Astellas, Pfizer, Schering Plough, Allergan and Rottapharm, SPE Pharma.

Professor Chris Chapple is a scientific advisor and researcher for Astellas, Pfizer, Novartis and Allergan.

Professor Alan Wein does not have any potential conflict of interest. 


\section{References}

1. Abrams P, Cardozo L, Fall M et al (2002) The standardisation of terminology of lower urinary tract function: report from the Standardisation Sub-committee of the International Continence Society. Neurourol Urodyn 21:167-178

2. Irwin DE, Milsom I, Hunskaar S et al (2006) Population-based survey of urinary incontinence, overactive bladder, and other lower urinary tract symptoms in five countries: results of the EPIC study. Eur Urol 50:1306-1315

3. Milsom I, Abrams P, Cardozo L et al (2001) How widespread are the symptoms of an overactive bladder and how are they managed? A population-based prevalence study. BJU Int 87:760-766

4. Stewart WF, Van Rooyen JB, Cundiff GW et al (2003) Prevalence and burden of overactive bladder in the United States. World J Urol 20:327-336

5. Chapple C, Artibani W, Cardozo L et al (2005) The role of urinary urgency and its measurement in the overactive bladder symptom syndrome: current concepts and future prospects. BJU Int 95(3):335-340

6. De Wachter S, Wyndaele J (2008) How sudden is a compelling desire to void? An observational cystometric study on the suddenness of this sensation. BJU Int 101(8):1000-1003
7. Cardozo L, Dixon A (2005) Increased warning time with darifenacin: a new concept in the management of urinary urgency. J Urol 173:1214-1218

8. Blaivas J, Panagopoulos G, Weiss J et al (2009) Two types of urgency. Neurourol Urodyn 28(3):188-190

9. Michel M, Chapple C (2009, this issue) Basic mechanisms of urgency: roles and benefits of pharmacotherapy. World J Urol 27. doi:10.1007/s00345-009-0446-5

10. Yamaguchi O, Aikawa K, Shishido K, Nomiya M (2009, this issue) Place of overactive bladder in male lower urinary tract symptoms. World J Urol 27. doi:10.1007/s00345-009-0470-5

11. Hanno P, Chapple C, Cardozo L (2009, this issue) Bladder pain syndrome/interstitial cystitis-a sense of urgency. World J Urol 27. doi:10.1007/s00345-009-0439-4

12. Yoshida M (2009, this issue) Urgency and the elderly population. World J Urol 27. doi:10.1007/s00345-009-0491-0

13. Dmochowski R, Fitzgerald M, Wyndaele JJ (2009, this issue) Measuring urgency in clinical practice. World J Urol 27. doi:10.1007/s00345-009-0467-0

14. Staskin D (2009, this issue) A call to action: the compelling need to advance the role of urgency. World J Urol 27. doi:10.1007/s00345009-0458-1 